\title{
Analysis of a multicentre cloud-based CT dosimetric database: preliminary results
}

\author{
Francesca Calderoni ${ }^{1}$, Federica Campanaro ${ }^{1}$, Paola Enrica Colombo ${ }^{1}$, Mauro Campoleoni ${ }^{2}$, Cristina De Mattia', \\ Federica Rottoli ${ }^{1}$, Giannicola Galetta ${ }^{3}$, Fabio Zucconi ${ }^{3}$, Andrea Pola ${ }^{4}$, Andrea Righini ${ }^{5}$, Fabio Triulzi ${ }^{6}$, \\ Angelo Vanzulli ${ }^{7 *}$ and Alberto Torresin ${ }^{1}$
}

\begin{abstract}
Background: To manage and analyse dosimetric data provided by computed tomography (CT) scanners from four Italian hospitals.

Methods: A radiation dose index monitoring (RDIM) software was used to collect anonymised exams stored in a cloud server. Since hospitals use different names for the same procedure, digital imaging and communications in medicine (DICOM) tags more appropriate to describe exams were selected and associated to study common names (SCNs) from a radiology playbook according to scan region and use of contrast media. Retrospective analysis was carried out to describe population and to evaluate dosimetric indexes and inaccuracies associated with SCNs.

Results: More than 400 procedures were clustered into 95 SCNs, but $78 \%$ of exams on adults were described with only 10 SCNs. Median values of dose-length product (DLP) and volumetric CT dose index (CTDI vol $_{\text {) }}$ for three analysed SCNs were in agreement with those previously published. The percentage of inaccuracies does not heavily affect the dosimetric analysis on the whole cloud, since variations in median values reached at most 8\%.

Conclusions: Implementation of a cloud-based RDIM software and related issues were described, showing the strength of the chosen playbook-based clustering and its usefulness for homogeneous data analysis. This approach may allow for optimisation actions, accurate assessment of the risk associated with radiation exposure, comparison of different facilities, and, last but not least, collection of information for the implementation of the 2013/59 Euratom Directive.
\end{abstract}

Keywords: Radiation dosage, Radiation exposure, Software, Tomography scanners ( $x$-ray computed), Radiation dose index monitoring software

\section{Key points}

- A radiation dose index monitoring software allowed to collect data on radiological exams and to store them in a cloud server.

- Clustering examinations through a radiological playbook is a good choice for data analysis.

- More than 400 computed tomography procedures were clustered into 95 study common names.

- Dose indexes for analysed study common names agree with those previously published.

\footnotetext{
* Correspondence: angelo.vanzulli@ospedaleniguarda.it

${ }^{7}$ Department of Radiology, ASST Grande Ospedale Metropolitano Niguarda,

Piazza Ospedale Maggiore 3, 20162 Milan, Italy

Full list of author information is available at the end of the article
}

- The inaccuracies of the system did not heavily affect dosimetric analysis on the whole cloud.

\section{Background}

The extensive use of computed tomography (CT) examinations in radiological diagnostics [1] caused an increasing attention to patient exposure and to the potential risk of carcinogenesis associated with relatively high radiation doses. Optimisation is mandatory to maintain the quality of the diagnostic information provided by the examination while seeking to reduce patient exposure to radiation to a level as low as reasonably achievable. The International Commission on radiological protection (ICRP) stated that a further optimisation can be obtained through collection of data from radiation dose structured reports in a digital format and through electronic data transfer from hospital and 
radiology information systems, providing data for large numbers of patients suitable for collection in a registry [2].

Hence, a useful way to monitor ionising radiation exposure caused by radiologic examinations is the adoption of a radiation dose index monitoring (RDIM) software. These software packages also allow to verify the compliance with the diagnostic reference levels (DRLs), facilitating surveys and improving the statistical strength of the analysis [35], optimise the exposures and compare different protocols or scanners. In order to analyse the exams performed with different CT scanners and in different hospitals in a consistent way, it is also necessary to cluster such large amount of data.

A great collection of data with these aims has been performed since 2011 by the American College of Radiology (ACR) that created the Dose Index Registry (DIR) [6]. The DIR includes more than 50 million CT exams transmitted automatically from scanners and arranged according to the RadLex ${ }^{\circ}$ playbook by the Radiological Society of North America [7, 8]. Kanal et al. [9] analysed the ten most common examinations within the DIR in order to develop DRLs and achievable doses as a function of patient size, obtaining values similar to those obtained by other countries for median-size patients. One of the limitations they highlighted are the unavoidable inaccuracies in examination clustering that may cause problems both in estimation of benchmark data and in comparison with them.

Parakh et al. [10] presented their experience with a radiation tracking software (RTS) for monitoring and comparing in relative terms cumulative patient effective doses and for calculating the average dose metrics, hence providing a global view of CT doses and defining a meaningful benchmark representing institutional DRLs. They stated that a critical step was to ensure that CT protocols on all scanners were consistently identified, a goal achieved by adopting the RadLex ${ }^{\circ}$ playbook.

Another publication by Parakh et al. [11] extended this kind of work to six medical institutions by collecting anonymised data from local servers into a single master server. The RTS allowed to perform analysis of different dose metrics, i.e., volumetric $\mathrm{CT}$ dose index $\left(\mathrm{CTDI}_{\mathrm{vol}}\right)$, dose-length product (DLP) and size-specific dose estimate and effective dose. To ensure a consistent analysis, a great effort was exerted in protocol matching using the RadLex $x^{\circ}$ playbook. Furthermore, the large number of CT scans reduced the effect of erroneous cases on the average dose metrics.

Also, Pyfferoen et al. [12] collected anonymised data from several hospitals through a RTS. They grouped the different protocol names under the reference anatomical regions according to available national DRLs in order to compare dose levels and scan lengths of standard adult CT examinations within three institutions and with national reference levels. Before the analysis, they performed a data check to eliminate, on the series level, those examinations in which the CT region did not match the clinical indication. Data checking at series level was performed also by MacGregor et al. [13] to verify the belonging to specific "master protocols" used for the clustering.

In addition to commercial systems, Boos et al. [14] implemented an in-house cloud-based CT RTS to automatically monitor dose data to make a comparison with national DRLs. Even if this study reported initial singlecentre results, the cloud-based approach enabled multicentre applications.

A cloud-based RDIM software was chosen by our group within a research project endorsed by Regione Lombardia, Italy. One of the aims of this project was to manage and analyse dosimetric data. A relevant task was to create a central database of dosimetric data, analysing exposure values collected through $13 \mathrm{CT}$ scanners installed in four different hospitals. The goal of this paper is to describe the feasibility of the cloud solution, presenting some preliminary results and discussing advantages and disadvantages of this cloud-based system.

\section{Methods}

The study was evaluated by our Institutional Review Board, and the requirement for informed consent was waived. The four hospitals involved were as follows: ASST Grande Ospedale Metropolitano Niguarda, Fondazione IRCCS Ca' Granda Ospedale Maggiore Policlinico, ASST Fatebenefratelli Sacco and Ospedale dei Bambini V. Buzzi. The first three are general hospitals, while the last one is paediatric.

To analyse the dosimetric archive, the associated large amount of data was clustered according to the RadLex playbook [15] as in the previously cited papers [9-11].

Data collected in 2017 were first analysed according to facilities, age and sex, to get descriptive statistics. In a second step, the distributions of dosimetric quantities were compared with values from the literature to check the strength of the cloud database. A systematic comparison with currently available reference levels was beyond the scope of this work. The same data were also used to assess the quantity of studies with series not matching with the original requirement and to evaluate their effect on dosimetric quantities.

\section{Description of the RDIM software and cloud server architecture}

The four hospitals were equipped with the RDIM software Bracco Injeneering's NEXO [DOSE $]^{\bullet}$ (Bracco Injeneering S.A., Lausanne, Switzerland), developed by PACSHealth, LLC, integrated with the different PACS of each hospital (Agfa, Fuji, Carestream). NEXO [DOSE] $]^{\circ}$ is a web-based software which collects patient information (age, sex, etc.) and dosimetric data.

Relevant data could be extracted from different sources: digital imaging and communications in medicine (DICOM) 


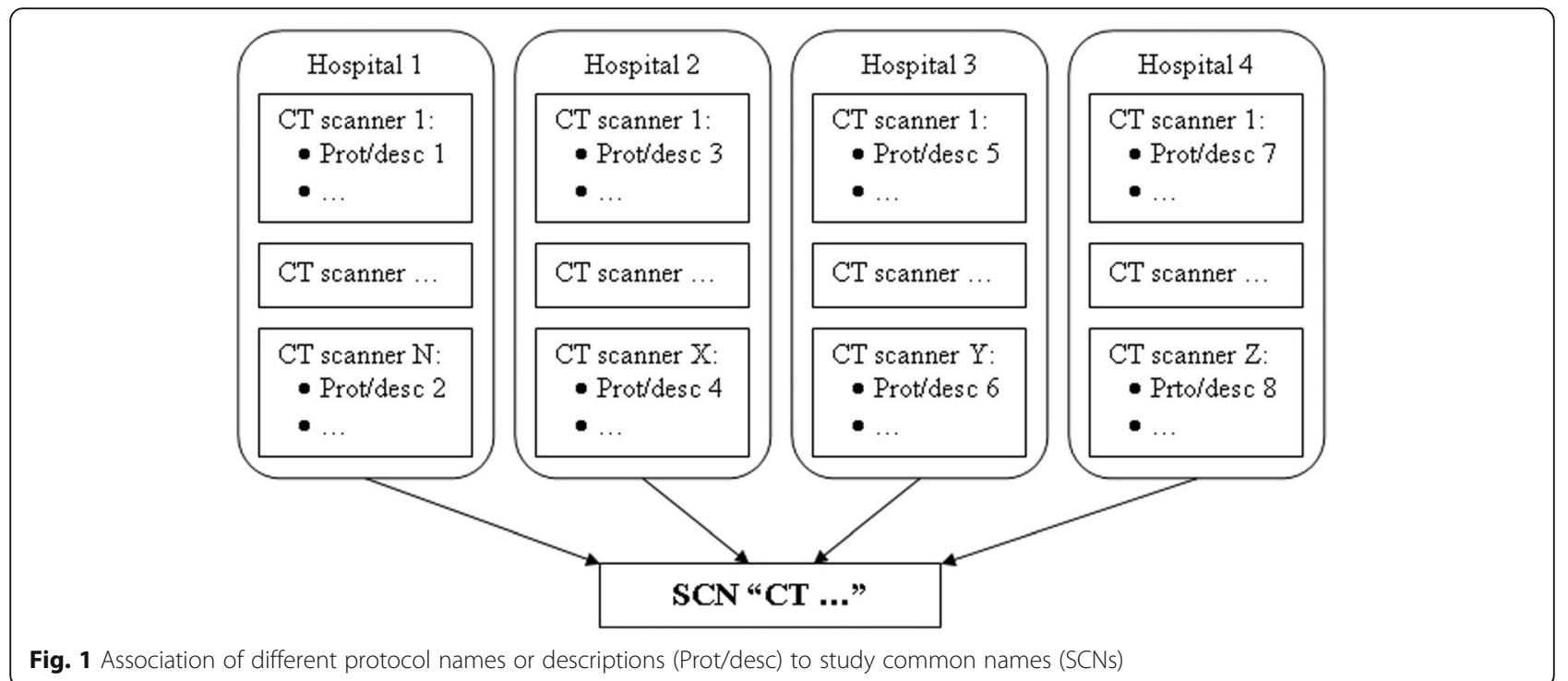

header (for each series), patient protocol (overall exam) and radiation dose structured report. Dealing with several CT scanners, the most suitable data sources were chosen for each device.

Data from the hospitals were collected both in local servers and in a cloud one.

Each individual institution has complied with its internal procedures to ensure the highest level of security and privacy of patient personal data. These procedures required the appointment of a person in charge of data processing, in this case, an external subject, the specification of access methods and the definition of the persons authorised to access the data, who undertake to behave in absolute confidentiality. In the case of the cloud server, data were anonymised prior to leaving the local site server following
DICOM PS3.15 [16] and Integrating the Healthcare Enterprise Radiation Exposure Monitoring RAD-63 profiles [17]. Patient data were removed, replaced, or modified in accordance with the reference DICOM standard. There were some exceptions, such as patient characteristics (age, sex, height, weight) and device information (facility, device, exposure parameters), for relevant data needed for statistical aims and analysis. Only the relevant data were transmitted, not the entire studies.

Finally, all data were collected in the Cloud NEXO [DOSE $^{\circ}$ Server - Microsoft Azure for Healthcare, in compliance with Health Insurance Portability and Accountability Act, International Organization for Standardization and European Union data protection directives, received via DICOM over transport layer security (TLS).

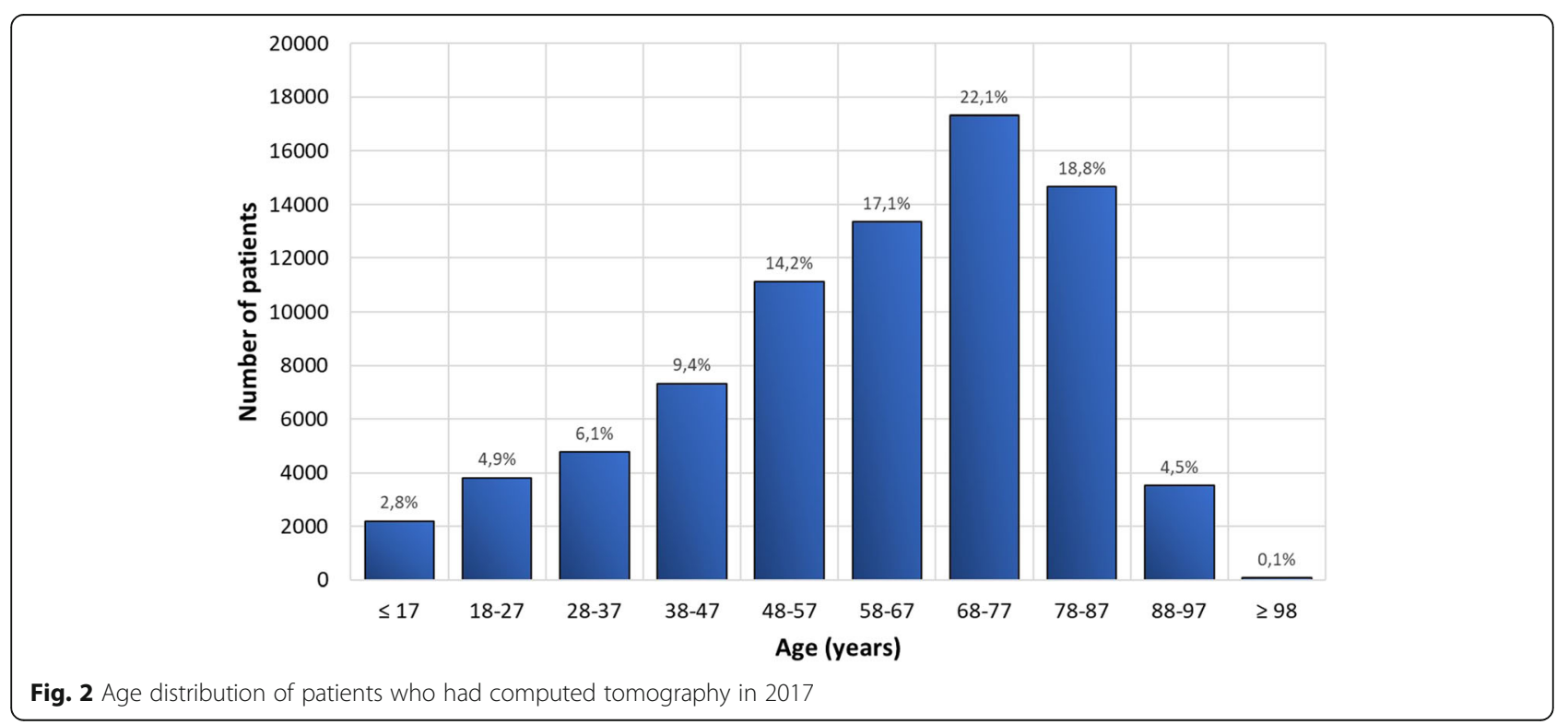



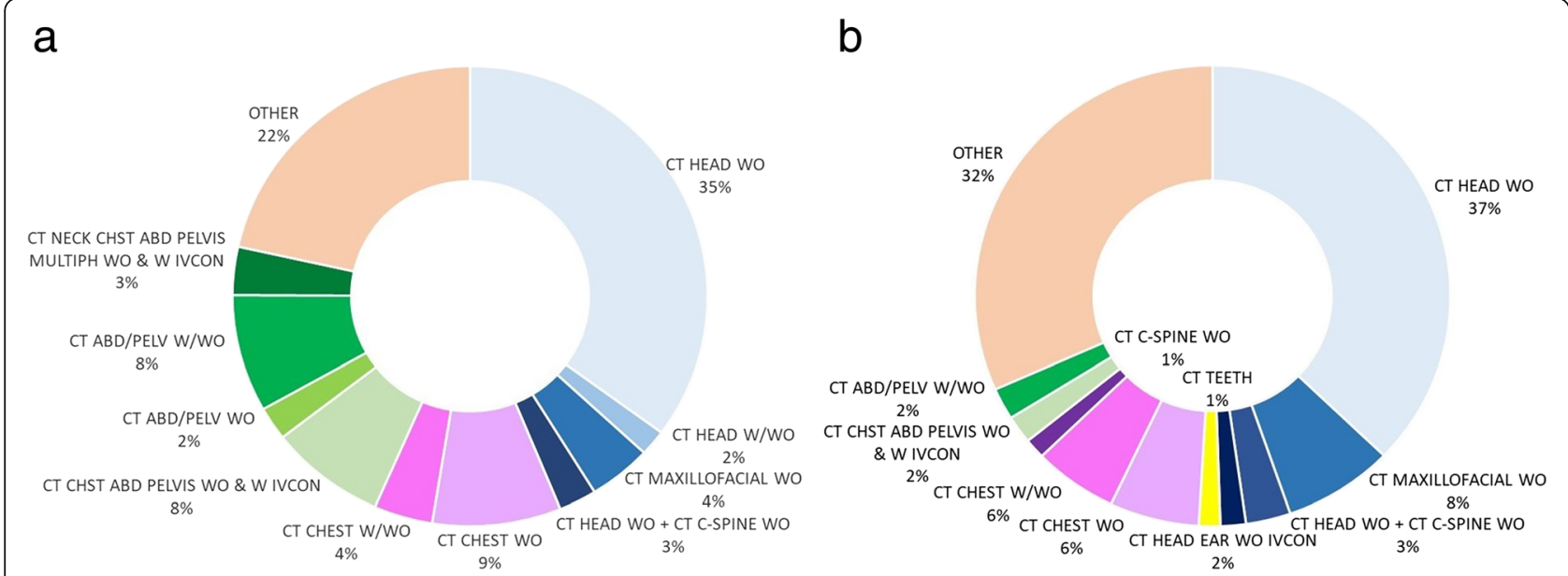

Fig. 3 Percentages of exams within the ten most common study common names in two different age ranges: 18-109 (a) and 0-17 (b). The main differences between the two distributions are the presence of many exams with a broad scan length including different anatomical regions in adult pie chart and the almost total absence of exams with contrast agent in paediatric ones. The majority of paediatric exams were in the head region compared to the adult distribution, with more "CT Maxillofacial WO" (traumas, sinusitis) and the addition of " $C T$ Teeth". The higher percentage of exams in the "OTHER" in the paediatric distribution could be related to the greater difficulty of the diagnosis in the absence of clinical history and with symptoms described by children

Each hospital could access the cloud database, and the software was able to report, for each exam, patient demographics (age, sex), and scan protocol information $\left(\mathrm{CTDI}_{\mathrm{vol}}, \mathrm{DLP}\right)$, with all the previously anonymised sensitive data. More detailed information relative to the single series could also be retrieved.

\section{Global descriptive analysis}

The analysis of the whole data stored in the cloud server can give a general description of the distribution of radiological exams among the population depending on facilities, age and sex.

Through NEXO [DOSE $]^{\circ}$, data were filtered according to facility, device, age, sex, and other characteristics in order to develop descriptive statistics of parameters relevant for the risk associated to radiation exposure, such as the percentages of males and females undergoing exams and the distribution of the number of patients as a function of age. Moreover, the number of exams of the different hospitals was tracked.

A first retrospective analysis regarding CT exams performed during 2017 was carried out.

\section{Detailed analysis of CT studies \\ Clustering}

The RadLex ${ }^{\circ}$ playbook is a project of the Radiological Society of North America [8] that provides a standardised system for naming radiological procedures. As in other studies [9-11], the RadLex ${ }^{\circ}$ playbook was used to cluster a great quantity of data for the subsequent analysis.

The arrangement of exams in homogeneous groups, according to scan region and acquisition task, is difficult due to the differences in types of CT scanner, radiology information systems (RIS) and picture archiving and communication system (PACS). We solved this problem using the radiological information stored in the different DICOM tags and inside the hospital reporting database. In two hospitals, the DICOM tag "study description" $(0008,1030)$ generated by the RIS was used; in another hospital, the same tag generated by the scanner was considered, whereas in the last one, the DICOM tag "protocol name" $(0018,1030)$ compiled with the scanner protocol name was chosen.

Table 1 Dose-length product (DLP) and volumetric computed tomography dose index $\left(\mathrm{CTDI}_{\mathrm{vo}}\right)$ for the study common name "CT Head WO": data from individual hospitals, total and reference values

\begin{tabular}{|c|c|c|c|}
\hline & $\begin{array}{l}\text { Number of exams/ } \\
\text { number of scanners }\end{array}$ & $\begin{array}{l}\text { DLP (mGy } \times \mathrm{cm}) \\
\text { Median (25th- } \\
\text { 75th percentile) }\end{array}$ & $\begin{array}{l}\text { CTDI }{ }_{\text {vol }} \text { (mGy) } \\
\text { Median (25th- } \\
\text { 75th percentile) }\end{array}$ \\
\hline $\begin{array}{l}\text { Hospital } \\
1\end{array}$ & $9,961 / 6$ & $853(798-898)$ & $54.8(53.3-57.8)$ \\
\hline $\begin{array}{l}\text { Hospital } \\
2\end{array}$ & $5,578 / 2$ & $1,131(1,004-1,339)$ & $60.4(51.3-60.9)$ \\
\hline $\begin{array}{l}\text { Hospital } \\
3\end{array}$ & $11,031 / 4$ & $1,017(830-1,022)$ & $58.6(54.8-58.7)$ \\
\hline $\begin{array}{l}\text { Hospital } \\
4\end{array}$ & $395 / 1$ & $1,121(969-1,468)$ & $68.4(60.5-76.0)$ \\
\hline Total & $26,965 / 13$ & $1,011(827-1,024)$ & $58.6(53.3-58.9)$ \\
\hline \multicolumn{2}{|c|}{ ISTISAN 17/33 [18] } & 1,382 & 69.0 \\
\hline \multicolumn{2}{|c|}{ RP 180 [19] } & $1,000(760-1,300)^{*}$ & $60(50-75)^{*}$ \\
\hline \multicolumn{2}{|c|}{ Canadian survey [20] } & $1,276(1,084-1,463)$ & $74.4(60.1-79.1)$ \\
\hline
\end{tabular}

*Most common value (range) 

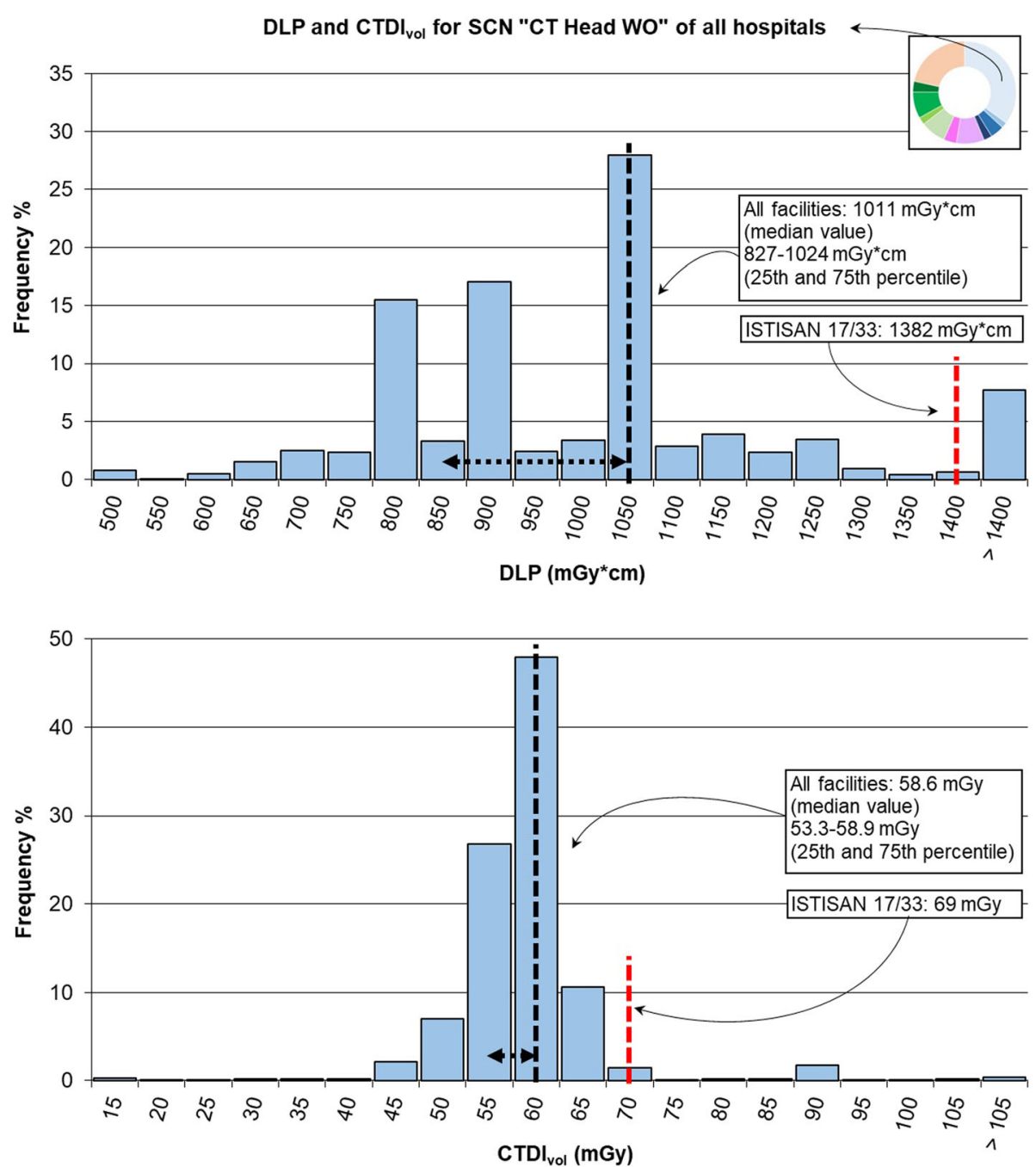

Fig. 4 Distribution of total dose-length product (DLP) and volumetric computed tomography dose index (CTDI ${ }_{\text {vol }}$ ) for study common name (SCN) " $C T$ Head WO" and comparison with values from ISTISAN 17/33 [18]. The median value and range between 25th and 75th percentile are shown in black dashed lines; the red dashed line reports the diagnostic reference level (DRL) provided by ISTISAN 17/33 [18]. The narrow shape of CTDIvol histogram points out similar values among scanners and highlights the use of fixed mAs values. The DLP histogram shows several peaks. On the one hand, the anatomical region was very similar from one patient to another; on the other hand, the scanners used different collimations, and therefore, the entire anatomical range is covered by a fixed and scanner-dependent number of acquisitions (axial mode). The final peak, more or less evident in all distributions (see the following Figs. 5, 6, 7, 8, and 9), is caused by the inclusion in the last class of values higher than the considered range

Table 2 Dose-length product (DLP) and volumetric computed tomography dose index ( $\left.C T D I_{\text {vol }}\right)$ for the study common name " $C T$ Chest WO": data from individual hospitals, total and reference values

\begin{tabular}{llll}
\hline & Number of exams/number of scanners & $\begin{array}{l}\text { DLP (mGy } \times \mathrm{cm}) \\
\text { Median (25th-75th percentile) }\end{array}$ & $\begin{array}{l}\text { CTDI } \\
\text { Mel (mGy) }\end{array}$ \\
\hline Hospital 1 & $3,513 / 5$ & $268(194-335)$ & $7.4(5.9-8.5)$ \\
Hospital 2 & $1,003 / 2$ & $323(232-468)$ & $7.1(5.9-8.8)$ \\
Hospital 3 & $2,026 / 4$ & $247(169-345)$ & $6.1(4.9-8.5)$ \\
Total & $6,542 / 11$ & $268(190-349)$ & $7.0(5.4-8.5)$ \\
ISTISAN 17/33 [18] & & 754 & 15.0 \\
RP 180 [19] & & $400(270-700)^{*}$ & $10(10-30)^{*}$ \\
Canadian survey [20] & $302(197-440)$ & $8.5(5.7-13.0)$ \\
\hline
\end{tabular}



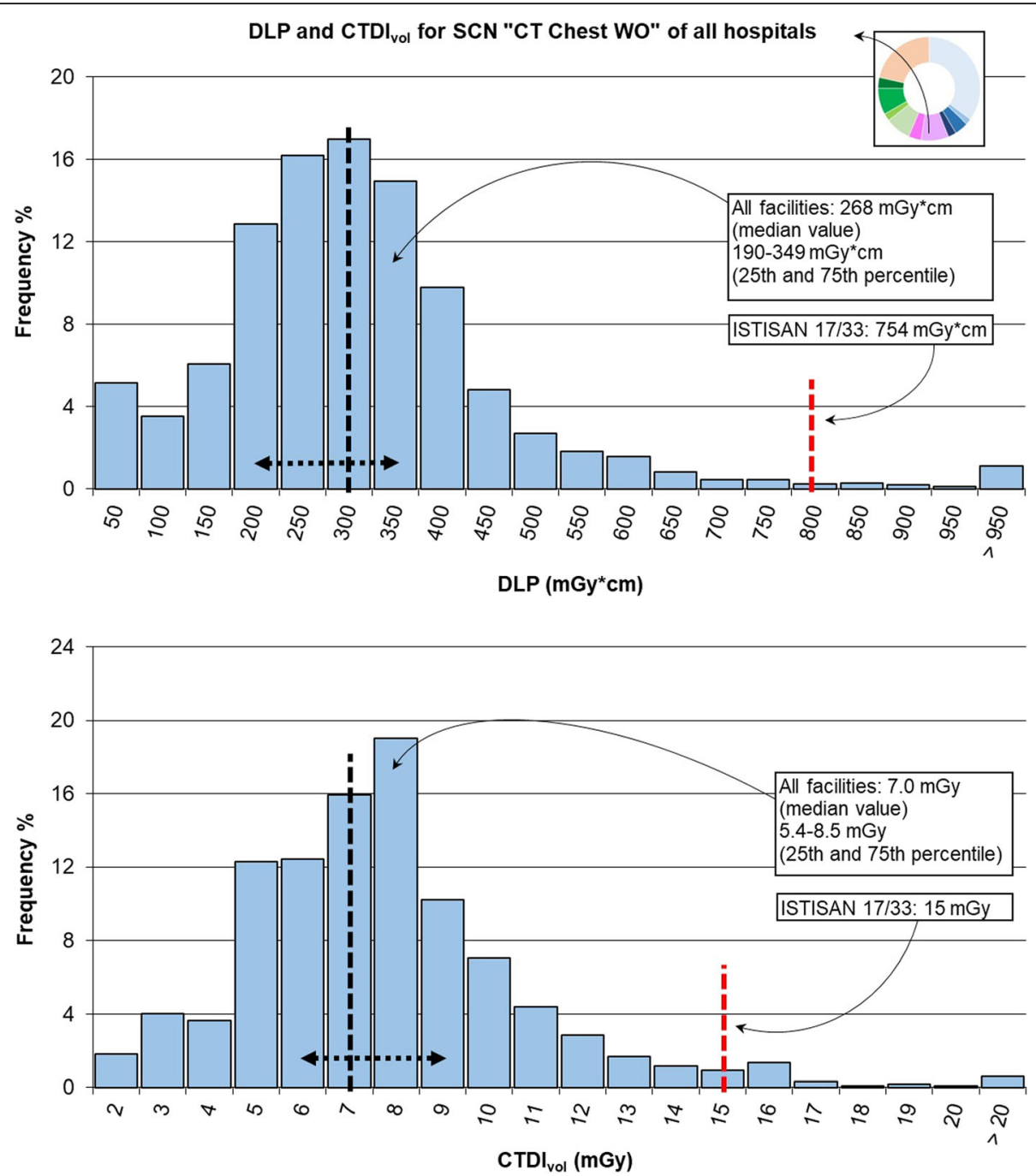

Fig. 5 Distribution of total dose-length product (DLP) and volumetric computed tomography dose index (CTDlvol) for study common name (SCN) "CT Chest WO" and comparison with values from ISTISAN 17/33 [18]. The median value and range between 25th and 75th percentile are shown in black dashed lines; the red dashed line reports the diagnostic reference level (DRL) provided by ISTISAN 17/33 [18]

Table 3 Dose-length product (DLP) and volumetric computed tomography dose index (CTDI $\left.{ }_{\text {vol }}\right)$ for the study common name "CT Abd/Pelv WO": data from individual hospitals, total and reference values

\begin{tabular}{llll}
\hline & $\begin{array}{l}\text { Number of exams/ } \\
\text { number of scanners }\end{array}$ & $\begin{array}{l}\text { DLP (mGy } \times \mathrm{cm}) \\
\text { Median (25th- } \\
\text { 75th percentile) }\end{array}$ & $\begin{array}{l}\text { CTDI } \\
\text { Median (mGy) } \\
\text { 75th percentile) }\end{array}$ \\
\hline $\begin{array}{l}\text { Hospital } \\
1\end{array}$ & $1,081 / 5$ & $543(425-743)$ & $10.4(8.2-13.1)$ \\
$\begin{array}{l}\text { Hospital } \\
2\end{array}$ & $326 / 2$ & $643(539-852)$ & $13.1(11.5-15.6)$ \\
$\begin{array}{l}\text { Hospital } \\
3\end{array}$ & $285 / 4$ & $590(455-771)$ & $11.8(10.1-14.3)$ \\
$\begin{array}{l}\text { Total } \\
\text { ISTISAN } \\
\text { 17/33 [18] }\end{array}$ & $569(446-769)$ & $11.2(8.9-14.0)$ \\
$\begin{array}{l}\text { Canadian } \\
\text { survey [20] }\end{array}$ & 920 & 15.0 \\
\hline
\end{tabular}

A study common name (SCN) from the RadLex playbook was associated to each description or protocol name to classify exams in a consistent way, according to scan region and use of contrast media, as represented in general terms in Fig. 1. Since the procedures within a RadLex label should have homogeneous exposure parameters, data organised in this way were used to analyse population and dosimetric quantities in a consistent way in order to evaluate the different radiological procedures.

Through NEXO [DOSE] $]^{\circ}$, data were filtered according to $\mathrm{SCN}$ and both studies and series were exported in Excel format. Files relative to studies include mean $\mathrm{CTDI}_{\mathrm{vol}}$ and total DLP, allowing to evaluate their distributions and to calculate median values, 25th and 75th percentiles. Variations of DLP with sex were also evaluated. 

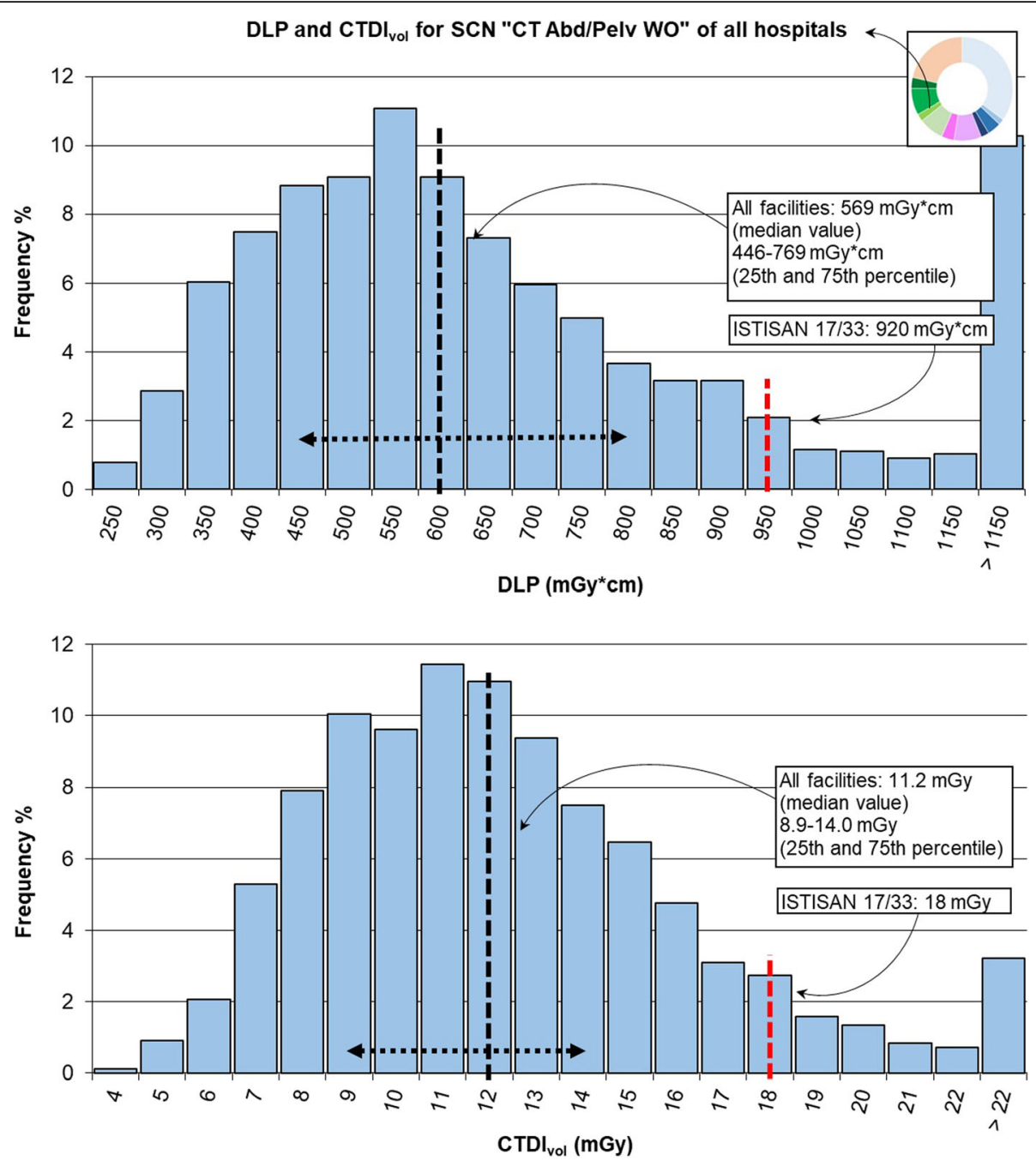

Fig. 6 Distribution of total dose-length product (DLP) and volumetric computed tomography dose index (CTDI vol $_{\text {I }}$ ) for study common name (SCN) "CT Abd/Pelv WO" and comparison with values from ISTISAN 17/33 [13]. The median value and range between 25th and 75th percentile are shown in black dashed lines; the red dashed line reports the diagnostic reference level (DRL) provided by ISTISAN 17/33 [18]

In the first phase of the study, 76,171 exams on adult patients (age range 18-109 years), the most frequent ones without administration of contrast agent (without contrast, "WO") were considered, in

Table 4 Ratio and percentage of exposures not in line (i.e., not in agreement with the study common name in terms of body region studied or use of contrast media) for the three analysed study common names: individual hospitals and total

\begin{tabular}{llll}
\hline & CT Head WO & CT Chest WO & CT Abd/Pelv WO \\
\hline Hospital 1 & $167 / 9,961(2 \%)$ & $42 / 3,513(1 \%)$ & $100 / 1,081(9 \%)$ \\
Hospital 2 & $1,269 / 5,578(23 \%)$ & $64 / 1,003(6 \%)$ & $29 / 326(9 \%)$ \\
Hospital 3 & $277 / 11,031(3 \%)$ & $442 / 2,026(22 \%)$ & $51 / 285(18 \%)$ \\
Hospital 4 & $0 / 395(0 \%)$ & NA & NA \\
Total & $1,713 / 26,965(6 \%)$ & $548 / 6,542(8 \%)$ & $180 / 1,692(11 \%)$ \\
\hline
\end{tabular}

Data in parentheses are percentages. NA Not available particular, those belonging to SCNs "CT Head WO" (35\%, 27,030 exams), "CT Chest WO" (9\%, 6,635 exams), and "CT Abd/Pelv WO" (2\%, 1,778 exams) in order to evaluate radiation exposure in different body regions. This analysis was performed at first with the whole data in the cloud, excluding those studies with coarse problems in the data transfer from the DICOM tag. In the case of "CT Chest WO" and "CT Abd/Pelv WO", only data from three hospitals were analysed since the few studies of the fourth one were not enough for statistical aims. CTDI ${ }_{\mathrm{vol}}$ and DLP values were depicted through histograms, including in the highest class values higher than the depicted range, but to check the strength of this clustering, only median values of dosimetric quantities were compared with recent DRLs, as indicated by ICRP 135 [2]. 


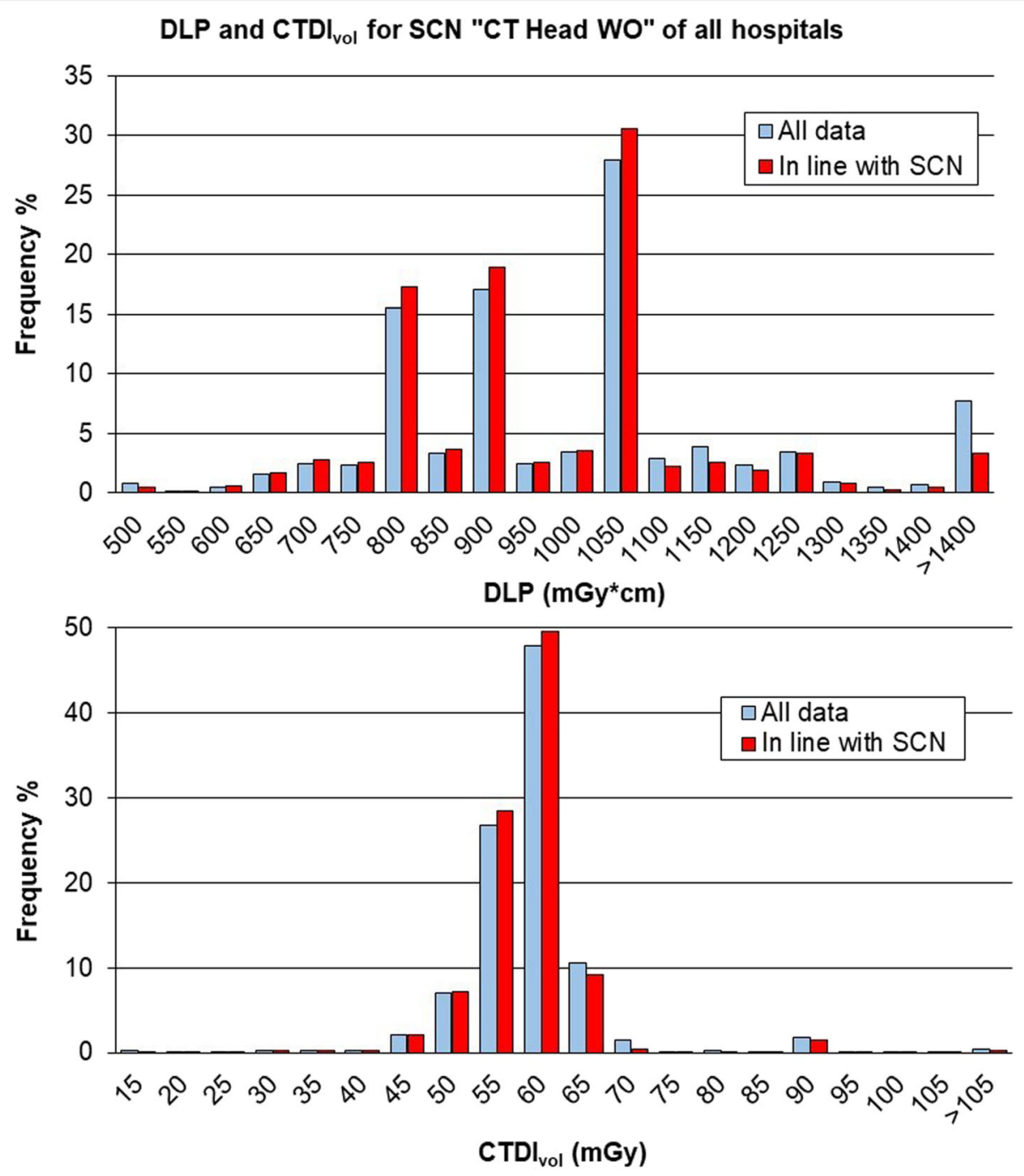

Fig. 7 Comparison between distributions of total dose-length product (DLP) and volumetric computed tomography dose index (CTDI $\mathrm{vol}_{\mathrm{vo}}$ ) of the whole data and those in line with the study common name (SCN) "CT Head WO", i.e., studies with all series belonging to that cluster

\section{Check of clustered data}

In the second phase of the study, a more accurate check of data within the SCNs was performed through series analysis following the methodology proposed in other publications $[12,13]$. Studies including series not in agreement with the SCN in terms of anatomical region or use of contrast media (exams not in line with the $\mathrm{SCN}$ ) were quantified and removed from the subsequent analysis. For example, within the SCN "CT Head WO" studies including series descriptions as "TorAdd 3.0 B40f", "C_Spine", "HeadAngio 0.75 H30f", and "Spine 2.0 B30s" were found and removed; the same for description as "HeadSeq 4.8 H31s" within the SCN "CT Chest WO".

The analysis of CTDI $\mathrm{vol}_{\mathrm{vol}}$ and DLP was thus repeated only with the studies in line with the SCNs in order to evaluate potential changes in median values and to test the strength of the database.

\section{Results}

Global descriptive analysis

All the 78,370 exams, including paediatric and adult ones, performed in the four hospitals were analysed. In particular, $49 \%$ of the exams were carried out at hospital $1,15 \%$ at hospital $2,34 \%$ at hospital 3 , and $2 \%$ at hospital 4 . The age distribution of the whole CT examinations is shown in Fig. 2. The large majority of exams are performed on adult patients ( $\geq 18$ years old), $97.2 \%$ against $2.8 \%$ of paediatrics $(<18$ years old), with predominance in the $68-77$ years old range. The distribution in terms of sex was as follows: $53.4 \%$ of patients were male whilst $46.6 \%$ were female.

\section{Detailed analysis of studies}

\section{Clustering}

More than $400 \mathrm{CT}$ procedures were clustered into 95 SCNs. Figure 3 shows the percentages of exams within 

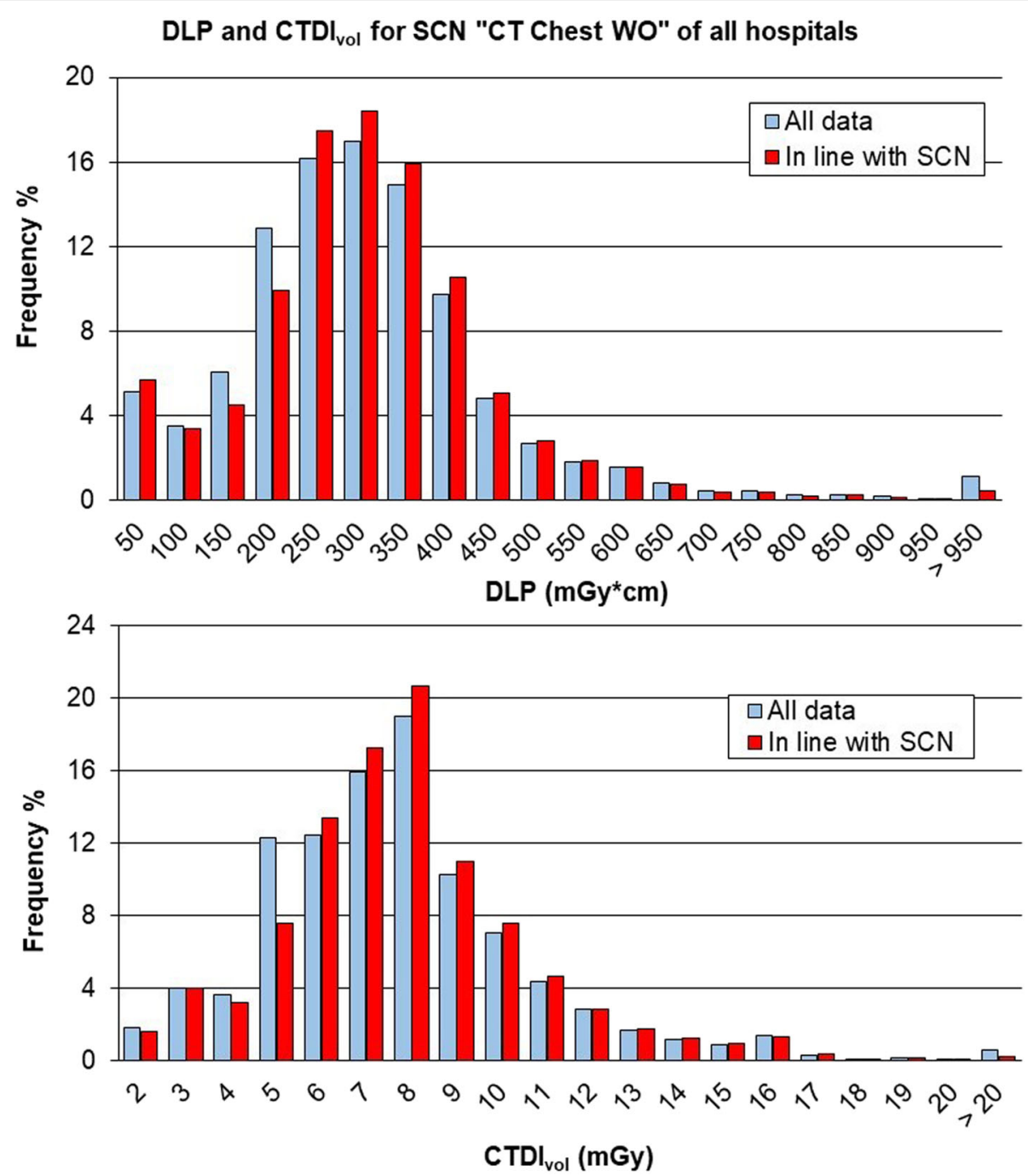

Fig. 8 Comparison between distributions of total dose-length product (DLP) and volumetric computed tomography dose index (CTDI $\mathrm{vol}_{\mathrm{vo}}$ ) of the whole data and those in line with the study common name (SCN) "CT Chest WO", i.e., studies with all series belonging to that cluster

the 10 most common SCNs, clustering a quantity of exams between 78\% (adult) and 68\% (paediatric) of the total, depicted for different age ranges: $18-109$ and 0 17. It is evident that the prevalent exam is the "CT Head WO", followed by SCNs with far fewer studies (less than $10 \%)$ such as "CT Chest WO", "CT Abd/Pelv W/WO", and "CT Chst Abd Pelvis WO \& W IVCON", which include all the remaining anatomical regions.

\section{Results for adults: "CT Head WO"}

Table 1 shows the median values of DLP and CTDI ${ }_{\mathrm{vol}}$ for SCN "CT Head WO" for the different hospitals $(26,965$ exams over 27,030). Hospital 1 had the lowest values of both DLP and CTDI ${ }_{\mathrm{vol}}$ while hospital 2 and hospital 4 had the highest values for DLP and CTDI ${ }_{\mathrm{vol}}$, respectively.

DLP for females was lower than that for males of about $5 \%$, mainly due to different scan lengths.
Figure 4 shows the distributions of DLP and CTDI $\mathrm{vol}_{\mathrm{vol}}$ for the whole data of the SCN "CT Head WO" related to the DRL values provided by the new Italian publication ISTISAN 17/33 [18].

Median values of radiation exposure with their 25th and 75th percentiles are also summarised in Table 1 (median DLP of $1011 \mathrm{mGy} \times \mathrm{cm}$; median CTDI ${ }_{\mathrm{vol}}$ of $58.6 \mathrm{mGy}$ ).

The total DLP distribution had a median value of $1,011 \mathrm{mGy} \times \mathrm{cm}$, close to $(1.1 \%$ higher $)$ the DRLs summarised by the 2014 European Commission Radiation Protection document 180 [19], which considers the most common value of $1,000 \mathrm{mGy} \times \mathrm{cm}$ and a range of $760-1$, $300 \mathrm{mGy} \times \mathrm{cm}$. The same was for the total $\mathrm{CTDI}_{\mathrm{vol}}$ of $58.6 \mathrm{mGy}$ which is $2.3 \%$ lower than the most common value of $60 \mathrm{mGy}$ with a range of 50-75 mGy.

The same comparison can be performed with values provided by recent publications on this topic, i.e., the US 

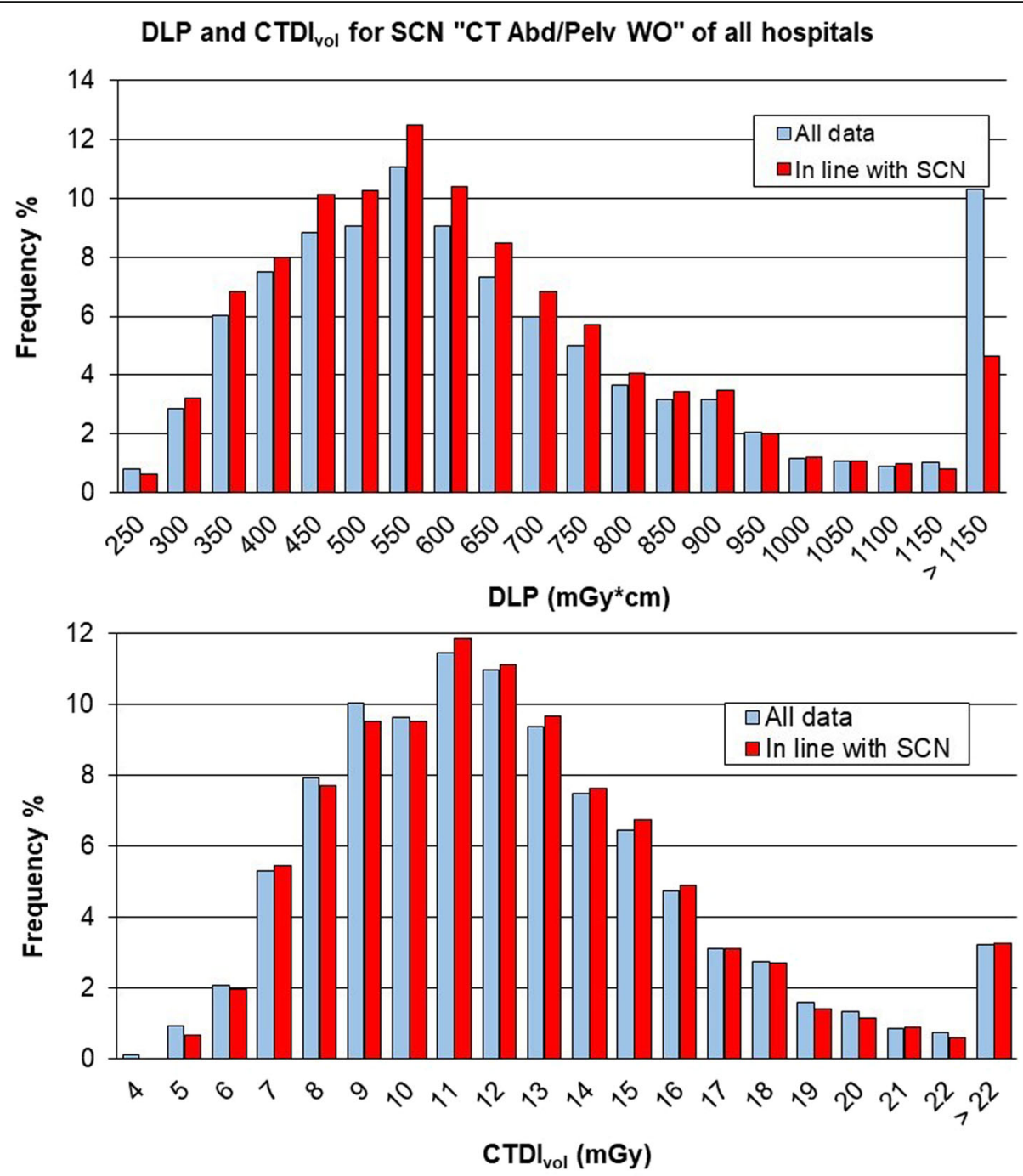

Fig. 9 Comparison between distributions of total dose-length product (DLP) and volumetric computed tomography dose index (CTDI vol $_{\text {) }}$ of the whole data and those in line with the study common name (SCN) "CT Abd/Pelv WO", i.e., studies with all series belonging to that cluster

Diagnostic Reference Levels and Achievable Doses [9] and the Canadian Computed Tomography Survey [20]. Our median values of DLP and CTDI ${ }_{\mathrm{vol}}$ were $5.1 \%$ and $4.6 \%$, respectively, higher than the US DRLs (expressed as 75th percentile for a median size patient), i.e., $962 \mathrm{mGy} \times \mathrm{cm}$ and $56 \mathrm{mGy}$, respectively. Compared to Canadian survey, our values were $20.8 \%$ and $21.2 \%$ lower, respectively, than those indicated in the subgroup "Adult head-helical/no contrast/fixed current" in terms of median value (25th percentile-75th percentile): DLP of $1,276 \mathrm{mGy} \times \mathrm{cm}$ (1, $084 \mathrm{mGy} \times \mathrm{cm}-1,463 \mathrm{mGy} \times \mathrm{cm})$, CTDI $_{\mathrm{vol}}$ of $74.4 \mathrm{mGy}$ (60.1 mGy-79.1 mGy).

\section{Results for adults: "CT Chest WO"}

The median values of DLP and $\mathrm{CTDI}_{\mathrm{vol}}$ for SCN "CT Chest WO" are shown in Table 2 (6,542 exams over 6,
635). In this case, hospital 3 has the lowest values of both the dosimetric indexes, whereas hospitals 2 and 1 have the highest values for DLP and $\mathrm{CTDI}_{\mathrm{vol}}$, respectively. Variations with sex showed values of DLP for females lower than that for males at about $19 \%$, mainly due to lower CTDI.

In the case of "CT chest WO" of Fig. 5, both the total DLP distribution and the CTDI $\mathrm{vol}_{\mathrm{v}}$ distribution have the shape of a gamma function as expected [1, 5]. The total DLP reported in Table 2 has a median value of $268 \mathrm{mGy} \times \mathrm{cm}, 33.0 \%$ lower than the DRL provided by the RP 180 [19] which considers the most common value of $400 \mathrm{mGy} \times \mathrm{cm}$ and a range of $270-700 \mathrm{mGy} \times \mathrm{cm}$. Also, in the case of the total $\mathrm{CTDI}_{\mathrm{vol}}$, the median value of $7.0 \mathrm{mGy}$ is $30.0 \%$ lower than the most common value of $10 \mathrm{mGy}$ with a range of $10-30$ mGy. 
Table 5 Dose-length product (DLP) and volumetric computed tomography dose index (CTDI vol) for "CT Head WO", "CT Chest WO" and "CT Abd/Pelv WO": data for exams in line with the study common name and for all exams

\begin{tabular}{|c|c|c|c|c|c|c|c|}
\hline & \multicolumn{3}{|c|}{ DLP all hospitals $(m G y \times c m)$} & \multicolumn{3}{|c|}{ CTDI $_{\text {vol }}$ all hospitals (mGy) } & \multirow{2}{*}{$\begin{array}{l}\text { Number } \\
\text { of } \\
\text { exams }\end{array}$} \\
\hline & Median & 25th percentile & 75th percentile & Median & 25th percentile & $\overline{75 \text { th percentile }}$ & \\
\hline \multicolumn{8}{|l|}{ CT Head WO } \\
\hline In line with SCN (A) & 933 & 798 & 1022 & 58.6 & 54.2 & 58.9 & 25,252 \\
\hline All data (B) & 1011 & 827 & 1024 & 57.8 & 53.3 & 58.9 & 26,965 \\
\hline$\Delta=(B-A) / B$ & $+7.7 \%$ & $+3.5 \%$ & $+0.2 \%$ & $-1.4 \%$ & $-1.7 \%$ & $0.0 \%$ & \\
\hline \multicolumn{8}{|l|}{ CT Chest WO } \\
\hline In line with SCN (A) & 274 & 205 & 349 & 7.2 & 5.7 & 8.6 & 5,994 \\
\hline All data (B) & 268 & 190 & 349 & 7.0 & 5.4 & 8.5 & 6,542 \\
\hline$\Delta=(B-A) / B$ & $-2.2 \%$ & $-7.9 \%$ & $0.0 \%$ & $-2.9 \%$ & $-5.6 \%$ & $-1.2 \%$ & \\
\hline \multicolumn{8}{|l|}{ CT Abd/Pelv WO } \\
\hline In line with SCN (A) & 553 & 438 & 710 & 11.2 & 9.0 & 14.0 & 1,512 \\
\hline All data (B) & 569 & 446 & 769 & 11.2 & 8.9 & 14.0 & 1,692 \\
\hline$\Delta=(B-A) / B$ & $+2.8 \%$ & $+1.8 \%$ & $+7.7 \%$ & $0.0 \%$ & $-1.1 \%$ & $0.0 \%$ & \\
\hline
\end{tabular}

The US DRLs [9] report a DLP of $443 \mathrm{mGy} \times \mathrm{cm}$ and a $\mathrm{CTDI}_{\mathrm{vol}}$ of $12 \mathrm{mGy}$. Hence, our results were $39.5 \%$ and $41.7 \%$ lower, respectively. The data provided by the Canadian computed tomography survey [20] for the subgroup "Adult chest-helical/no contrast/dose reduction" were DLP $302 \mathrm{mGy} \times \mathrm{cm} \quad(197 \mathrm{mGy} \times \mathrm{cm}-440 \mathrm{mGy} \times$ $\mathrm{cm}), \mathrm{CTDI}_{\mathrm{vol}} 8.5 \mathrm{mGy}(5.7 \mathrm{mGy}-13.0 \mathrm{mGy})$. Hence, our results were $11.3 \%$ and $17.6 \%$ lower, respectively.

\section{Results for adults: "CT Abd/Pelv WO"}

The median values of DLP and CTDI ${ }_{\mathrm{vol}}$ for $\mathrm{SCN}$ "CT Abd/Pelv WO" are reported in Table 3 (1,692 exams over 1,778). As for the SCN "CT head WO", the lowest values are those of hospital 1 while hospital 2 has again the highest values. Variations with sex show values of DLP for females lower than that for males of about 3\%, due both to lower CTDI and scan length.

The total DLP and CTDI ${ }_{\text {vol }}$ distributions for the SCN "CT Abd/Pelv WO" of Fig. 6 had median values of 569 mGy $\times$ $\mathrm{cm}$ and $11.2 \mathrm{mGy}$, respectively, summarised in Table 3 .

A comparison with the values from the European Commission Radiation Protection document 180 [19] was not possible since this document provides two different values for the abdomen and pelvis. Compared to the US DRLs [9] (DLP equal to $781 \mathrm{mGy} \times \mathrm{cm}$ and $\mathrm{CTDI}_{\mathrm{vol}}$ to $16 \mathrm{mGy}$ ), our values were $27.1 \%$ and $30.0 \%$ lower, respectively. These results, DLP $10.3 \%$ higher and $\mathrm{CTDI}_{\mathrm{vol}}$ 13.2\% lower, are also comparable with the data obtained by the Canadian Computed Tomography Survey [20]: DLP $516 \mathrm{mGy} \times \mathrm{cm}(349 \mathrm{mGy} \times \mathrm{cm}-735 \mathrm{mGy} \times$ $\mathrm{cm}), \mathrm{CTDI}_{\mathrm{vol}} 12.9 \mathrm{mGy}(8.6 \mathrm{mGy}-17.6 \mathrm{mGy})$.

\section{Check of clustered data}

The series analysis shows that some irradiation events do not belong to the considered SCN. This means that the study changes compared to the prescription, but it can be justified by the necessity of more information in relation to the initial clinical question. The percentage of exposures not in line with the analysed SCNs is different for single hospitals, as summarised in Table 4 in comparison with the whole data. Considering the whole data from the four facilities, the exposures in line with the SCN are always about 90\%, as reported in Table 4.

The distributions of DLP and CTDI ${ }_{\mathrm{vol}}$ obtained with these data are represented in Figs. 7, 8 and 9 in comparison with the previous overall distributions.

These comparisons are also summed up in Table 5. Even if the percentage of exams not in line with SCN for single hospitals reaches the $23 \%$, the median values of dosimetric quantities for whole data vary for a few percent only, 7.7\% at most (DLP of CT Head WO in Table 5).

\section{Discussion}

The aim of this study was to evaluate advantages and disadvantages of a cloud-based CT dosimetric database, which represents the state of the art in terms of data collection for further optimisation.

These patient demographics and scan protocol information can be used for optimisation processes within each hospital and to compare the different facilities as well as for evaluation of risk due to patients' exposure to ionising radiations, e.g., the distributions in terms of age and sex are necessary for a detailed risk analysis [1]. 
The dosimetric database was implemented overcoming the problems of dealing with hundreds of protocols from 13 CT scanners used in four hospitals with different RIS/PACS. Clustering through RadLex ${ }^{\circ}$ playbook turned out to be a good choice for the subsequent analysis of data allowing data collection in a more homogeneous way. Our results show that by using RadLex ${ }^{\circ}$ playbook, more than $400 \mathrm{CT}$ procedures have been clustered in just 95 SCNs, but only ten SCNs described almost 80\% of the exams. The prevalent exam is the "CT Head WO", confirming the trend of other publications [1], which represents almost $40 \%$ of the studies. Despite the large use of CT in this anatomical region, these exposures are associated with a lower risk [21, 22].

As a preliminary analysis, the data for adult patients from the three main SCNs without the use of intravenous contrast agent were explored. The median values of DLP and CTDI $_{\mathrm{vol}}$ of all hospitals are well below the national DRL levels [18]. They are also close to European DRLs summarised by document 180 [19] and to the recent US Diagnostic Reference Levels and Achievable Doses [9] and Canadian Computed Tomography Survey [20]. The RDIM software was already in use in the hospitals even before 2017, and during these years, analysis was performed to allow optimisation of protocols, reducing patient exposure.

This preliminary analysis shows some differences between scanners of different hospitals as well, probably linked to the technology of the single devices, differences in protocol settings or specific requirements of the radiologists.

The presence of different RIS/PACS systems, CT scanners and protocols in each hospital, makes the implementation of this database more difficult and increases the probability of inaccuracies. A more detailed analysis of the single series present in the cloud highlights the presence of data that do not belong to the particular $\mathrm{SCN}$. This can be explained by differences in the management of the reconciliation between the required exam and the performed one, due to clinical needs or emergencies. In particular, storage and medical reporting of neurologic examinations are organised in different ways. For example, in hospital 1, head CTs are completely disjoined by other anatomical regions.

However, variations in median values of DLP and $\mathrm{CTDI}_{\mathrm{vol}}$ reach at most $8 \%$. Because of the great amount of data, some inaccuracies were expected. Nevertheless, they do not heavily affect the dosimetric analysis obtained through the database, allowing the simpler use of the whole data without previous reviews.

In conclusion, the implementation of a dosimetric database requires considerable efforts to configure each scanner and to cluster the CT protocols of different hospitals; RadLex ${ }^{\circ}$ playbook has proved to be an excellent tool for the comparison of homogeneous examinations. In this way, it is possible to optimise acquisitions obtaining a fair compromise between image quality and reduction of patient exposure. This process can be improved when several facilities, with expertise and skills in different clinical areas, populate the database and establish relevant benchmarks.

\section{Abbreviations \\ CT: Computed tomography; $C T D I_{\text {vol: }}$ Volumetric computed tomography dose index; DICOM: Digital imaging and communications in medicine; DIR: Dose index registry; DLP: Dose-length product; DRL: Diagnostic reference level; RDIM: Radiation dose index monitoring; RTS: Radiation tracking software; SCN: Study common name}

\section{Acknowledgements}

The authors wish to thank Marco Ciboldi, Enrico Cuoco, Giancarlo Oliva, Gianni Origgi, Giorgio Orsi, Gianluca Viganò, and Giuseppe Vighi for their contributions to this project.

\section{Authors' contributions}

FC and FeC analysed and interpreted the data and drafted the work. PEC, $M C, G G$, and FZ designed the work and interpreted the data. CDM and FR designed and revised the work. AP, AR, FT, AV, and AT revised the work. All authors read and approved the final manuscript.

\section{Funding}

This study received funding by Direzione Generale Sanità, Regione Lombardia, Call per la ricerca indipendente 2010-2013.

\section{Availability of data and materials}

The datasets used and analysed during the current study are available from the corresponding author on reasonable request.

\section{Ethics approval and consent to participate}

The study was evaluated by the Institutional Review Board (ASST Grande Ospedale Metropolitano Niguarda), and the requirement for informed consent was waived.

\section{Consent for publication}

Not applicable.

\section{Competing interests}

The authors declare that they have no competing interests.

\section{Author details}

${ }^{1}$ Department of Medical Physics, ASST Grande Ospedale Metropolitano Niguarda, Piazza Ospedale Maggiore 3, 20162 Milan, Italy. ${ }^{2}$ Medical Physics Unit, Fondazione IRCCS Ca' Granda Ospedale Maggiore Policlinico, Via Pace 9, 20122 Milan, Italy. ${ }^{3}$ Medical Physics, ASST Fatebenefratelli Sacco, via G.B. Grassi 74, 20157 Milan, Italy. ${ }^{4}$ Department of Energy, Politecnico di Milano, via La Masa 34, 20156 Milan, Italy. ${ }^{5}$ Pediatric Radiology and Neuroradiology Unit, Children's Hospital V. Buzzi, Via Castelvetro 32, 20154 Milan, Italy. ${ }^{6}$ Department of Neuroradiology, Fondazione IRCCS Ca' Granda Ospedale Maggiore Policlinico, Via Pace 9, 20122 Milan, Italy. ${ }^{7}$ Department of Radiology, ASST Grande Ospedale Metropolitano Niguarda, Piazza Ospedale Maggiore 3, 20162 Milan, Italy.

Received: 20 February 2019 Accepted: 3 June 2019

Published online: 16 July 2019

\section{References}

1. Pola A, Corbella D, Righini A et al (2018) Computed tomography use in a large Italian region: trend analysis 2004-2014 of emergency and outpatient CT examinations in children and adults. Eur Radiol 28:2308-2318. https:// doi.org/10.1007/s00330-017-5225-x

2. Vañ E, Miller DL, Martin CJ et al (2017) ICRP publication 135: diagnostic reference levels in medical imaging. Ann ICRP 46:1-144. https://doi.org/10. $1177 / 0146645317717209$

3. Liang CR, Chen PXH, Kapur J, Ong MKL, Quek ST, Kapur SC (2017) Establishment of institutional diagnostic reference level for computed tomography with 
automated dose-tracking software. J Med Radiat Sci 64:82-89. https://doi.org/10. 1002/jmrs.210

4. Treier R, Aroua A, Verdun FR, Samara E, Stuessi A, Trueb PR (2010) Patient doses in CT examinations in Switzerland: implementation of national diagnostic reference levels. Radiat Prot Dosimetry 142:244-254. https://doi.org/10.1093/rpd/ncq279

5. Palorini F, Origgi D, Granata C, Matranga D, Salerno S (2014) Adult exposures from MDCT including multiphase studies: first Italian nationwide survey. Eur Radiol 24:469-483. https://doi.org/10.1007/s00330-013-3031-7

6. American College of Radiology (2018) National Radiology Data Registry (NRDR). American College of Radiology's Dose Index Registry (DIR) website. Available via https:/hrdrsupport.acr.org/support/solutions/articles/11000028993

7. Langlotz CP (2006) RadLex: a new method for indexing online educational materials. Radiographics 26:1595-1597. https://doi.org/10.1148/rg.266065168

8. Radiological Society of North America (2018) RadLex playbook website. Available via http://playbook.radlex.org/playbook/SearchRadlexAction

9. Kanal KM, Butler PF, Sengupta D, Bhargavan-Chatfield M, Coombs LP, Morin RL (2017) U.S. diagnostic reference levels and achievable doses for 10 adult CT examinations. Radiology 284:120-133. https://doi.org/10.1148/radiol. 2017161911

10. Parakh A, Kortesniemi M, Schindera ST (2016) CT radiation dose management: a comprehensive optimization process for improving patient safety. Radiology 280:663-673. https:/doi.org/10.1148/radiol.2016151173

11. Parakh A, Euler A, Szucs-Farkas Z, Schindera ST (2017) Transatlantic comparison of $C T$ radiation doses in the era of radiation dose-tracking software. AJR Am J Roentgenol 209:1302-1307. https://doi.org/10.2214/AJR. 17.18087

12. Pyfferoen L, Mulkens TH, Zanca F, De Bondt T, Parizel PM, Casselman JW (2017) Benchmarking adult $\mathrm{CT}$-dose levels to regional and national references using a dose-tracking software: a multicentre experience. Insights Imaging 8:513-521. https://doi.org/10.1007/s13244-017-0570-5

13. MacGregor K, Li I, Dowdell T, Gray BG (2015) Identifying institutional diagnostic reference levels for $C T$ with radiation dose index monitoring software. Radiology 276:507-517. https://doi.org/10.1148/radiol.2015141520

14. Boos J, Meineke A, Rubbert C et al (2016) Cloud-based CT dose monitoring using the DICOM structured report: fully automated analysis in regard to national diagnostic reference levels. Rofo 188:288-294. https://doi.org/10. 1055/s-0041-108059

15. Wang KC, Patel FB, Vyas B et al (2017) Use of radiology procedure codes in health care: the need for standardization and structure. Radiographics 37 : 1099-1110. https://doi.org/10.1148/rg.2017160188

16. DICOM Standards Committee (2019) DICOM PS3.15 2019b - security and system management profiles. Chapter $\mathrm{E}$ attribute confidentiality profiles. Available via http://dicom.nema.org/medical/dicom/current/output/chtml/ part15/chapter_E.html

17. Integrating the Healthcare Enterprise (2013) IHE radiology technical framework, volume 3: transactions, revision 12.0. Available via https:// www.ihe.net/uploadedFiles/Documents/Radiology/IHE_RAD_TF_Rev12. 0_Vol3_FT_2013-09-06.pdf

18. Padovani $R$, Compagnone G, D'Ercole L et al (2017) Livelli diagnostici di riferimento nazionali per la radiologia diagnostica e interventistica. Istituto Superiore di Sanità, Roma Rapporti ISTISAN 17/33. Available via http://old iss.it/binary/publ/cont/17_33_web.pdf

19. European Commission (2014) Radiation protection n. 180 - diagnostic reference levels in thirty-six European countries - part 2/2. Avaliable via https://ec.europa.eu/energy/sites/ener/files/documents/RP180\%20part2.pdf

20. Health Canada (2016) Canadian computed tomography survey - national diagnostic reference levels. Available via https:/www.canada.ca/en/healthcanada/services/publications/health-risks-safety/canadian-computedtomography-survey-national-diagnostic-reference-levels.html
21. Sodickson A, Baeyens PF, Andriole KP et al (2009) Recurrent CT, cumulative radiation exposure, and associated radiation-induced cancer risks from $\mathrm{CT}$ of adults. Radiology 251:175-184. https://doi.org/10.1148/radiol.2511081296

22. Børretzen I, Lysdahl KB, Olerud HM (2007) Diagnostic radiology in Norway trends in examination frequency and collective effective dose. Radiat Prot Dosimetry 124:339-347. https://doi.org/10.1093/rpd/ncm204

\section{Publisher's Note}

Springer Nature remains neutral with regard to jurisdictional claims in published maps and institutional affiliations.

\section{Submit your manuscript to a SpringerOpen ${ }^{\circ}$ journal and benefit from:}

- Convenient online submission

- Rigorous peer review

- Open access: articles freely available online

- High visibility within the field

- Retaining the copyright to your article

Submit your next manuscript at $\boldsymbol{\nabla}$ springeropen.com 